\title{
Principais características da atuação dos profissionais de enfermagem da Atenção Primária em Saúde na assistência ao paciente soropositivo
}

\section{Main characteristics of the performance of nursing professionals in Primaty Health Care in the care of seropositive patients}

Luana Maria Lima Feitosa ${ }^{1}$, Sandra Rodrigues da Silva Rosa ${ }^{2}$, Leidiany Souza Silva $^{3}$, Rogério Carvalho de Figueredo ${ }^{4}$, Rafael Souza Silva ${ }^{5}$

\section{RESUMO}

Apesar dos avanços no tratamento da Síndrome da Imunodeficiência Adquirida (AIDS) e no diagnóstico do seu agente etiológico, o Vírus da Imunodeficiência Humana (HIV), a assistência de enfermagem oferecida aos pacientes soropositivos ainda requer mudanças que contemplem a debilitação que afeta o organismo dos pacientes, os estigmas sociais e a prevenção mais eficaz da doença. $O$ objetivo do estudo foi identificar as principais características expressadas pelos enfermeiros e técnicos de enfermagem da Atenção Primária em Saúde (APS), diante da prestação de cuidados ao paciente soropositivo. Trata-se de uma pesquisa de campo descritiva, segundo os pressupostos do método quantitativo, realizada em uma Unidade Básica de Saúde do município do interior do Estado do Tocantins, com profissionais de enfermagem. Dentre os principais resultados, percebeu-se que a maioria dos participantes não conhece sobre às formas de controle da infecção pelo HIV/AIDS, e não receberam treinamento para a realização de testes rápidos. Quanto à realização de campanhas para prevenção do HIV, a maioria dos pesquisados afirmaram desenvolver ações educativas, porém disseram ter dificuldades. Portanto, os profissionais de enfermagem da APS apresentam um papel importante na prevenção da infecção pelo HIV e na prestação da assistência aos pacientes soropositivos. Entretanto, ainda existe um déficit de conhecimentos quanto HIV/AIDS, por parte da equipe de enfermagem; evidenciando a necessidade de atualizações relacionadas a temática.

Palavras-chave: Assistência de enfermagem. Paciente Soropositivo. Atenção Primária em Saúde.

\section{ABSTRACT}

Despite advances in the treatment of Acquired Immunodeficiency Syndrome (AIDS) and in the diagnosis of its etiologic agent, the Human Immunodeficiency Virus (HIV), the nursing care offered to HIV-positive patients still requires changes that contemplate the debilitation that affects the organism of patient, social stigmas and more effective disease prevention. The objective of the study was to identify the main characteristics expressed by nurses and nursing technicians in Primary Health Care (PHC), when providing care to HIV-positive patients. It is a descriptive field research, according to the assumptions of the quantitative method, carried out in a Basic Health Unit in the city of the interior of the State of Tocantins, with nursing professionals. Among the main results, it was noticed that the majority of the participants do not know about the ways of controlling HIV / AIDS infection, and did not receive training to perform rapid tests. As for HIV prevention campaigns, most respondents said they developed educational actions, but said they had difficulties. Therefore, PHC nursing professionals play an important role in preventing HIV infection and in providing care to HIV-positive patients. However, there is still a lack of knowledge regarding HIV / AIDS, on the part of the nursing team; highlighting the need for updates related to the theme.
Acadêmica do curso de bacharel em Enfermagem do IESC FAG.

E-mail: Iuhmaria87@hotmail.com

Acadêmica do curso de bacharel em Enfermagem do IESC FAG.

Enfermeira, especialista em Saúde Pública. Professora do curso de bacharel em Enfermagem do IESC FAG.

Enfermeiro, mestre em Ciências da Saúde. Professor do curso de bacharel em Enfermagem do IESC FAG.

Enfermeiro, especialista em Urgência e Emergência. Professor do curso de bacharel em Enfermagem do IESC FAG.

Key-words: Nursing care. Seropositive patient. Primary Health Care. 


\section{INTRODUÇÄO}

O termo "AIDS" é conceituado como uma patologia relacionada à contaminação pelo Vírus da Imunodeficiência Humana (HIV), que por sua vez, pode ocasionar a debilitação do organismo, pois se instala no sistema imunológico do indivíduo ocasionando infecção que pode danificar gradativamente o sistema de defesa, levando a vulnerabilidade do paciente a diversas afecções. ${ }^{1,2}$

Desde a descoberta e o surgimento das primeiras discussões sobre o HIV/AIDS, existe preconceito, discriminação, medo e insegurança em relação ao contato direto com o paciente contaminado, além de ausência de conhecimentos abrangentes sobre o assunto. Deste modo, esses sentimentos também estão presentes nos profissionais da saúde, quanto ao que se refere a prestação da assistência ao paciente soropositivo. ${ }^{3}$

Os profissionais que atuam na Atenção Primária em Saúde possuem uma função primordial na diminuição da exposição ao HIV/AIDS, pois a mesma envolve ações de caráter pessoal e comunitário, incluindo atividades de prevenção e promoção à saúde dos pacientes. Ressalta - se, entre estes, os enfermeiros que convivem com momentos delicados como o diagnóstico e a assistência ao portador da doença, tendo papel fundamental na atenção ao soropositivo. ${ }^{4,5}$

A assistência de enfermagem ao paciente soropositivo inclui desde o conhecimento da patologia em si, abrangendo suas características, diagnóstico e tratamento, ao acolhimento dos fatores psicológicos, físicos e espirituais. Entre eles respectivamente, o preconceito social devido à incompreensão da infecção; o histórico de doenças imunológicas que favorecem a ação do vírus no organismo; a crença e a fé pelo seu grande potencial colaborativo ao tratamento. Para isso, é essencial que o profissional de enfermagem mantenha o elo com o paciente e sua família, com o intuito de compreender o contexto a qual o paciente está inserido, sua percepção sobre a doença, assim como as interferências sociais e espirituais que possam influenciar no tratamento da doença. ${ }^{2}$

De acordo com o Código de Ética dos Profissionais de Enfermagem (CEPE), existem princípios que são considerados primordiais para subsidiar o trabalho desta categoria, os quais incluem a promoção, prevenção e recuperação da saúde do ser humano de forma eficaz, em nível individual, familiar e comunitário. ${ }^{6}$ 
$\mathrm{Na}$ atualidade, ainda existem estigmas no atendimento ao usuário infectado, principalmente quanto as formas de transmissão e o preconceito social relacionado aos portadores do vírus. Entre os principais estigmas podemos citar a abordagem pelo profissional ao usuário soropositivo, que ainda é realizada de forma preconceituosa, e não segue os preceitos da humanização do cuidar. Alguns estudos evidenciam que profissionais da saúde ainda discriminam os usuários soropositivos quanto a cor e raça, pelo sexo, pela condição social ou falta de dinheiro, pela orientação sexual, pela profissão ou ocupação. ${ }^{14}$

Os profissionais de enfermagem apresentam fragilidades e dificuldades na assistência a esses pacientes, uma vez que os enfermeiros não estão completamente preparados no que tange ao desenvolvimento de métodos para enfrentar a situação instável das pessoas que são portadoras do HIV/AIDS. ${ }^{7}$

Neste contexto, percebe-se que a assistência de enfermagem possui grande relevância no que se refere à atenção ao paciente portador do HIV e sua realidade, incluindo em assuntos relacionados ao contágio; detecção prévia; seguimento da terapia antirretroviral; aceitação do público-alvo às estratégias e programas públicos; bem como o fortalecimento da autonomia e do cuidado individual. ${ }^{5}$

Outro fator existente está relacionado ao preconceito direcionado ao soropositivo, por ser visto como o responsável pela propagação da patologia, ocasionando a discriminação deste por parte da comunidade, da própria família e do enfermeiro durante a prestação da assistência à saúde. Portanto, esse profissional necessita possuir conhecimento científico essencial para o atendimento às necessidades psicológicas do indivíduo com HIV/AIDS. ${ }^{8}$

O objetivo desta pesquisa foi identificar as principais características expressadas pelos enfermeiros e técnicos de enfermagem da Atenção Primária em Saúde (APS), diante da prestação de cuidados ao paciente soropositivo.

\section{MATERIAIS E METODOS}

Trata-se de uma pesquisa de campo descritiva, segundo o pressuposto do método quantitativo. A pesquisa de campo foi realizada com profissionais de enfermagem, de ambos os gêneros, da Atenção Primária em Saúde, do Município de Presidente Kenedy, uma cidade na região norte do Estado do Tocantins, localizado as margens da 
BR-153, há aproximadamente $200 \mathrm{~km}$ da capital Palmas. A amostra foi constituída de quatro (04) enfermeiros e sete (07) técnicos de enfermagem da Unidade Básica de Saúde que concordaram voluntariamente em participar deste trabalho e se encaixaram nos critérios de inclusão da pesquisa.

O estudo segue os preceitos éticos da resolução 466/12 do Conselho Nacional de Saúde sendo submetido à Plataforma Brasil e aprovado pelo Comitê de Ética em Pesquisa do Hospital de Doenças Tropicais - Universidade Federal do Tocantins, com o parecer consubstanciado número 3.671.467 e CAAE: 20700819.3.0000.8102.

Os critérios de inclusão desta pesquisa foram profissionais da enfermagem, entre eles os enfermeiros e os técnicos de enfermagem, com idade mínima de 22 anos e máxima de 60 anos, que atuam nas Unidades Básicas de Saúde a mais de um semestre e consentiram voluntariamente em cooperar com a pesquisa e assinaram o "Termo de Consentimento Livre e Esclarecido (TCLE)". Foram excluídos, os demais profissionais da Atenção Primária e todos aqueles que não estavam de acordo com os critérios de inclusão preestabelecidos ou não concordaram em participar da pesquisa.

A coleta de dados ocorreu através da aplicação de um questionário semiestruturado contendo questões objetivas e dissertativas, baseado na versão final do questionário para avaliar o controle do HIV/AIDS na Atenção Básica sob a ótica dos profissionais da saúde, elaborado por Silva, Castro, Pereira e Oliveira; retirado do artigo intitulado "Questionário para avaliação das ações de controle do HIVIAIDS na Atenção Básica”. ${ }^{10}$

Os dados coletados foram analisados utilizando o método quantitativo, através de cálculos de porcentagem simples, representados em gráficos e tabelas, e fundamentados teoricamente com auxílio de referenciais bibliográficos, com o objetivo de compreender os resultados encontrados e compará-los aos resultados de outros autores.

\section{RESULTADOS E DISCUSSÁO}

Os resultados da pesquisa estão apresentados através de tabelas e gráficos seguidos de suas respectivas discussões com a literatura atual. E está organizado em dois capítulos sendo o primeiro com a caracterização dos participantes e o segundo com as principais adversidades entre os profissionais de enfermagem da APS acerca do HIV/AIDS. 


\section{CARACTERIZAÇÃO DOS PARTICIPANTES DA PESQUISA}

Tabela 1: As características dos participantes da pesquisa.

\begin{tabular}{|c|c|c|}
\hline ESCOLARIDADE & $n^{\circ}$ & $\%$ \\
\hline Enfermeiros Graduados & 3 & $27,2 \%$ \\
\hline Técnicos de Enfermagem & 7 & $63,7 \%$ \\
\hline Enfermeiros Especialistas & 1 & $9,1 \%$ \\
\hline TOTAL & 11 & $100 \%$ \\
\hline \multicolumn{3}{|l|}{ TEMPO DE ATUAÇÃO } \\
\hline 6 meses a 5 anos & 3 & $27,3 \%$ \\
\hline 5 a 10 anos & 2 & $18,2 \%$ \\
\hline 10 a 20 anos & 1 & $9,1 \%$ \\
\hline Mais que 20 anos & 5 & $45,4 \%$ \\
\hline TOTAL & 11 & $100 \%$ \\
\hline \multicolumn{3}{|l|}{ IDADE } \\
\hline $22-30$ anos & 4 & $36,4 \%$ \\
\hline $31-40$ anos & 1 & $9,1 \%$ \\
\hline $41-50$ anos & 2 & 18,1 \\
\hline Mais que 50 anos & 4 & $36,4 \%$ \\
\hline TOTAL & 11 & $100 \%$ \\
\hline \multicolumn{3}{|l|}{ SEXO } \\
\hline Feminino & 10 & $90,9 \%$ \\
\hline Masculino & 1 & $9,1 \%$ \\
\hline TOTAL & 11 & $100 \%$ \\
\hline \multicolumn{3}{|l|}{ RELIGIÃO } \\
\hline Católicos & 6 & $54,5 \%$ \\
\hline Evangélicos & 5 & $45,5 \%$ \\
\hline TOTAL & 11 & $100 \%$ \\
\hline
\end{tabular}

Fonte: Elaboração própria dos autores com base em dados coletados em pesquisa (2019).

Entre os profissionais pesquisados, $63,7 \%$ possuem o curso técnico de enfermagem, $27,2 \%$ são graduados em enfermagem e apenas $9,1 \%$ possuem especialização. (Tabela 1).

A idade variou, sendo $36,4 \%$ com idade $\geq$ de $22-30$ anos ou mais que 50 anos, $18,1 \%$ estão entre 41 à 50 anos e apenas 9,1\% apresentam-se com idade de 31 e 40 anos (Tabela 1). Em relação ao tempo de atuação, 27,3\% atuavam como profissionais há quase dois anos, 18,2\% há mais ou menos dez anos, 9,1\% entre 10 e 20 anos; e 45,4\% exercem a profissão há mais de vinte anos. (Tabela 1). A idade e o tempo de atuação são aspectos relevantes no exercício da enfermagem, uma vez que, a realização das incumbências da profissão requer ânimo, vitalidade, capacidade e resistência física. ${ }^{24}$ 
De acordo com o Conselho Federal de Enfermagem (COFEN), a profissão de enfermagem é na atualidade constituída no Brasil por um percentual de $20 \%$ de enfermeiros e $80 \%$ de técnicos e auxiliares de enfermagem. ${ }^{23}$

No que se refere ao sexo, $90,9 \%$ eram do sexo feminino e $9,1 \%$ do sexo masculino. Isto se justifica pelo fato de que, desde o surgimento da profissão de enfermagem, ocorre uma maior prevalência do sexo feminino. Embora nos últimos anos tenha tido um aumento na quantidade de homens que ingressaram na profissão de enfermagem, as mulheres ainda continuam dominando a profissão. ${ }^{25}$ Segundo o COFEN, a profissão de enfermagem é majoritariamente feminina, pois é constituída por um quantitativo de $84,6 \%$ de mulheres. ${ }^{23}$ Quanto à religião, $54,5 \%$ são católicos e $45,5 \%$ evangélicos.

\section{PRINCIPAIS ADVERSIDADES ENTRE OS PROFISSIONAIS DE ENFERMAGEM DA ATENÇÃO PRIMÁRIA QUANTO AO HIV/AIDS}

Dentre os profissionais de enfermagem pesquisados, $72,73 \%$ afirmaram ter alcance a materiais didáticos provenientes do Ministério da Saúde (MS), relacionados aos cuidados com as Infecções Sexualmente Transmissíveis (IST's). Enquanto 27,27\% dos participantes responderam não ter alcance. Quanto a ter compreensão sobre os assuntos abordado nos Manuais do MS, 72,73\% disseram "sim" e 27,27\% responderam "não" (Gráfico 1).

Gráfico 1. O acesso a informações por meio de materiais didáticos.

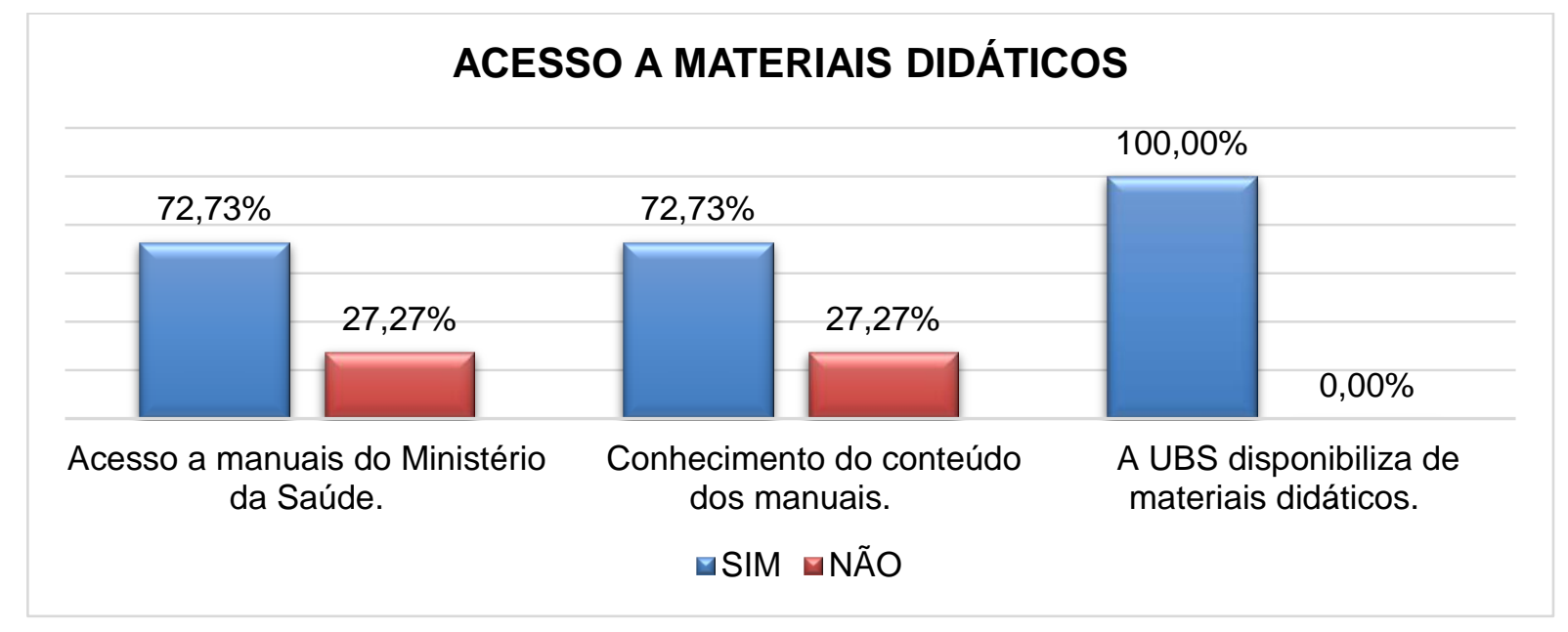

Fonte: Elaboração própria das autoras com base em dados coletados em pesquisa (2019). 
Quando questionados se a UBS oferta instrumentos didáticos para o desenvolvimento de atividades educativas, todos os profissionais $(100 \%)$ responderam "sim". Porém, quando interrogados sobre quais seriam os materiais didáticos ofertados, alguns dos pesquisados mostraram desconhecimento sobre a definição dos referidos materiais já citados, uma vez que alguns mencionaram "preservativos" como resposta para a questão. Vale ressaltar que materiais didáticos são instrumentos educativos utilizados com o intuito de ensinar, ou seja, de disseminar conhecimentos (como livros, folder, panfletos, manuais, entre outros).

Apesar de a maioria dos profissionais pesquisados afirmarem possuírem alcance e compreensão sobre os materiais ofertados pelo Ministério da Saúde, percebe-se por meio do estudo que os enfermeiros e técnicos de enfermagem demonstram deficiência de conhecimento abrangente sobre a temática. Portanto, é visível a necessidade de atualização desses profissionais como forma de proporcionar um melhor atendimento aos pacientes da Unidade Básica de Saúde. ${ }^{26}$

No princípio da epidemia do HIV/AIDS, o acolhimento de pacientes soropositivos, ocasionou preocupações nos profissionais de enfermagem, provocando complexidade relativa à prestação de cuidados. O temor, a discriminação, a aflição, a pouca compreensão sobre a doença e a incerteza diante da assistência diária eram frequentes, por perceberem que a patologia além de poder ser transmitida, também pode levar à morte. Por isso, os enfermeiros e técnicos de enfermagem necessitam de capacitação relacionada ao âmbito psíquico e fisiológico da sua atuação para que possa dominar seus sentimentos e proporcionar um atendimento de qualidade aos pacientes. ${ }^{11}$

Dos participantes, $81,82 \%$ afirmaram não ter recebido nenhum treinamento sobre métodos de controle da infecção pelo HIV/AIDS nos últimos cinco anos. E apenas 18,18\% disseram ter recebido atualização. É extremamente necessário que os profissionais de enfermagem participem de projetos de educação permanente, uma vez que é por meio dessas vivências que é possível adquirir atualizações e novos conhecimentos que poderão auxiliar de forma significativa na prestação da assistência de forma eficaz e satisfatória. ${ }^{26}$

Em relação ao desenvolvimento de campanhas educativas no território de cobertura da UBS, voltadas para a transmissão de informações sobre os riscos para a contaminação por HIV; $81,82 \%$ dos participantes disseram desenvolver campanhas, enquanto que $18,18 \%$ afirmaram não realizar. Quando perguntados sobre quais 
campanhas eram desenvolvidas, a maioria respondeu "palestras nas escolas" e "campanhas educativas no mês de dezembro" (mês de prevenção da AIDS) (Gráfico 2).

Quanto a execução de campanhas de educação em saúde relacionadas a prevenção do HIV/AIDS no território de abrangência da UBS; 45,45\% afirmaram desenvolver ações educativas e 54,55\% responderam não realizar. Quando questionados, novamente, sobre quais seriam essas campanhas realizadas, grande parte dos que responderam "sim", citaram "palestras" (Gráfico 2).

Gráfico 2. A realização de campanhas informativas relacionadas à prevenção do HIV/AIDS.

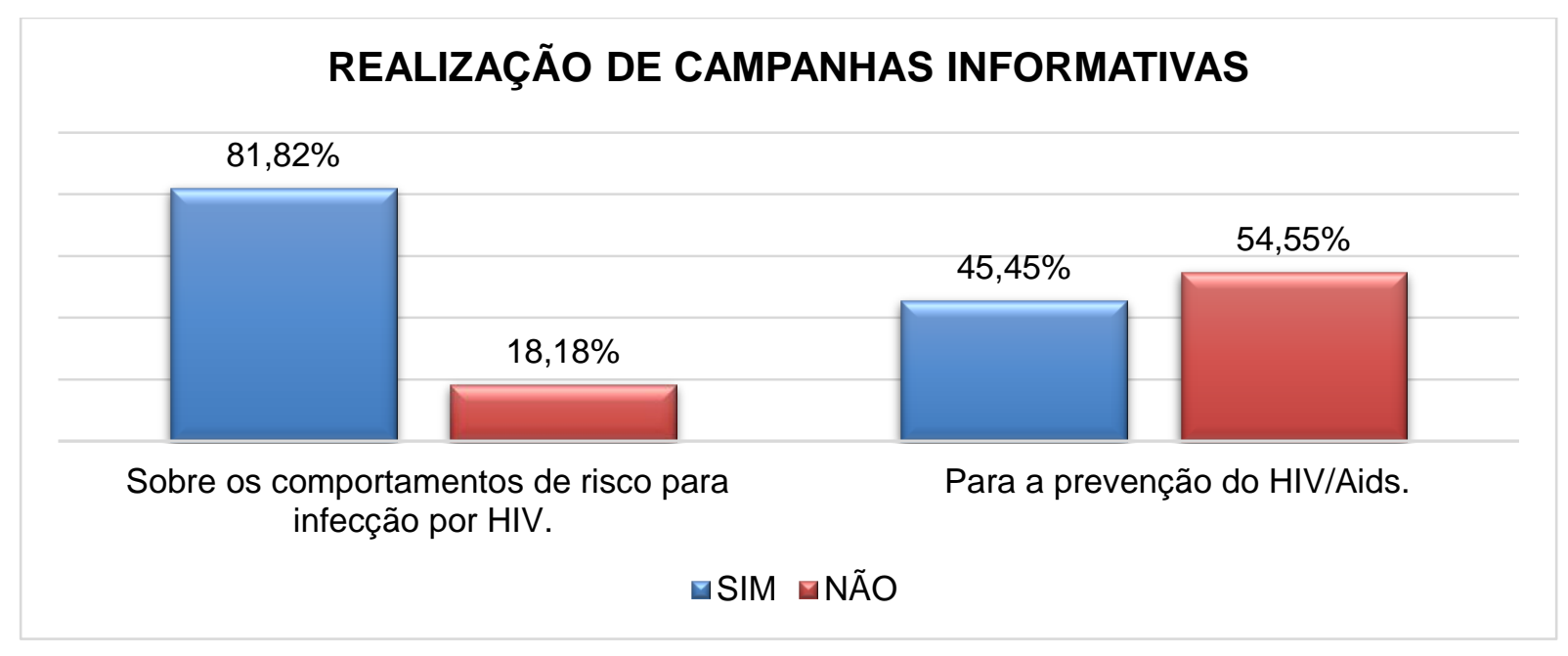

Fonte: Elaboração própria das autoras com base em dados coletados em pesquisa (2019).

O desenvolvimento de campanhas educativas é de suma importância para promover a conscientização da população sobre os fatores de risco e as medidas de prevenção da infecção pelo HIV, pois durante a realização das campanhas poderão ser utilizados métodos direcionados à discussão sobre a temática e esclarecimento de possíveis dúvidas dos pacientes. ${ }^{27}$

No que se refere a comprovação de gravidez por pacientes portadoras da infecção pelo HIV; todos os profissionais de enfermagem da pesquisa afirmaram iniciar a consulta de pré-natal o mais breve possível.

Todos os enfermeiros e técnicos de enfermagem da pesquisa (100\%) confirmaram que as mulheres grávidas diagnosticadas com HIV/AIDS, que foram encaminhadas a estabelecimentos de saúde de média e alta complexidade, também são assistidas pela Unidade Básica de Saúde. 
A assistência de enfermagem às gestantes diagnosticadas com HIV/AIDS é fundamental, uma vez que nestes casos se faz necessária uma atenção especial no prénatal, pois por meio do acompanhamento adequado da gestante soropositiva, é possível diminuir o risco de Transmissão vertical, e consequentemente evitar o aumento do percentual de crianças contaminadas pelo HIV. ${ }^{28}$

Quando questionados se a UBS possui uma estrutura física apropriada para o desenvolvimento de ações de educação em saúde, todos os profissionais pesquisados responderam "sim" (Gráfico 3).

No que corresponde a realização de atividades de educação em saúde relacionadas ao HIV/AIDS; os participantes da pesquisa foram perguntados se essas atividades são executadas sem dificuldades, onde 45,45\% responderam "sim" e 54,55\% responderam "não" (Gráfico 3).

Gráfico 3. O desenvolvimento de ações educativas pelos profissionais de enfermagem.

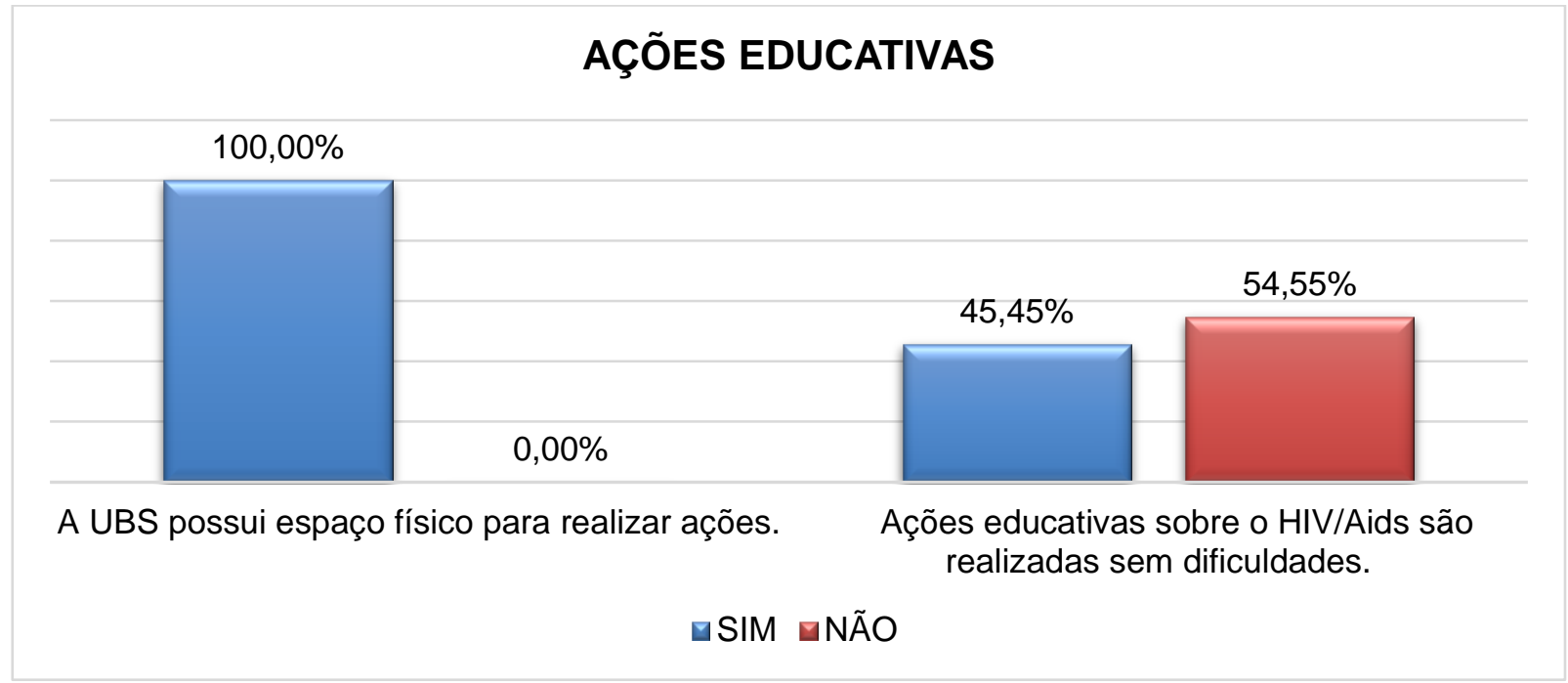

Fonte: Elaboração própria das autoras com base em dados coletados em pesquisa (2019).

De fato, o desenvolvimento de ações educativas é muito importante para a ampliação dos conhecimentos dos pacientes, porém, a estrutura física adequada e a organização da Unidade Básica de Saúde são fundamentais para a realização dessas ações de forma eficaz e satisfatória. ${ }^{26}$

A realização de ações de educação em saúde pelos profissionais de enfermagem é essencial, principalmente no que se refere a atividades educativas direcionadas a prevenção do HIV, pois o enfermeiro é visto como um profissional que pode contribuir 
com a propagação de informações e conhecimentos aos pacientes, familiares e coletividade. ${ }^{26}$

Convém ressaltar que a prestação do cuidado ao indivíduo com HIV/AIDS não é considerada uma ação simples de ser desenvolvida no ponto de vista dos enfermeiros, pois os obstáculos são criados quanto à realização do ato de cuidar. Desta forma, é relevante a adesão de um posicionamento ético, objetivando levar os pacientes soropositivos a usufruir deste cuidado por meio das intervenções realizadas, que serão úteis para a promoção da qualidade de vida. ${ }^{22}$

Todos os enfermeiros e técnicos de enfermagem pesquisados afirmaram que os testes rápidos e sorológicos são disponibilizados aos parceiros de pacientes portadores da infecção pelo HIV/AIDS. Quanto ao desenvolvimento de busca ativa dos parceiros sexuais dos pacientes identificados com HIV/AIDS, 100\% dos participantes da pesquisa afirmaram realizar.

Em casos de diagnóstico positivo para o HIV, é primordial a execução da busca ativa dos parceiros sexuais do paciente contaminado, uma vez que se faz necessária à realização do aconselhamento e dos testes para o HIV, com o intuito de verificar uma possível infecção do parceiro (a). Tudo isso deve ser feito com muita atenção, mantendo o sigilo e a privacidade dos envolvidos. ${ }^{29}$

Em relação a distribuição de preservativos para a população fora do estabelecimento de saúde; $90,91 \%$ dos pesquisados afirmaram realizar essa distribuição da camisinha, enquanto 9,09\% responderam "não" (Gráfico 4). Quando questionados em quais locais acontecia essa distribuição de preservativos, citaram lugares variados como: "Próximo a casas noturnas", "nas ruas", "nas proximidades da BR 153" e "na recepção da UBS".

Entre os profissionais de enfermagem que participaram da pesquisa, $63,64 \%$ disseram ofertar explicações relacionadas ao uso do preservativo no momento da distribuição. Por outro lado, 36,36\% afirmaram não realizar orientações (Gráfico 4). 
Gráfico 4. A entrega de preservativos feminino e masculino na Atenção Primária em Saúde.

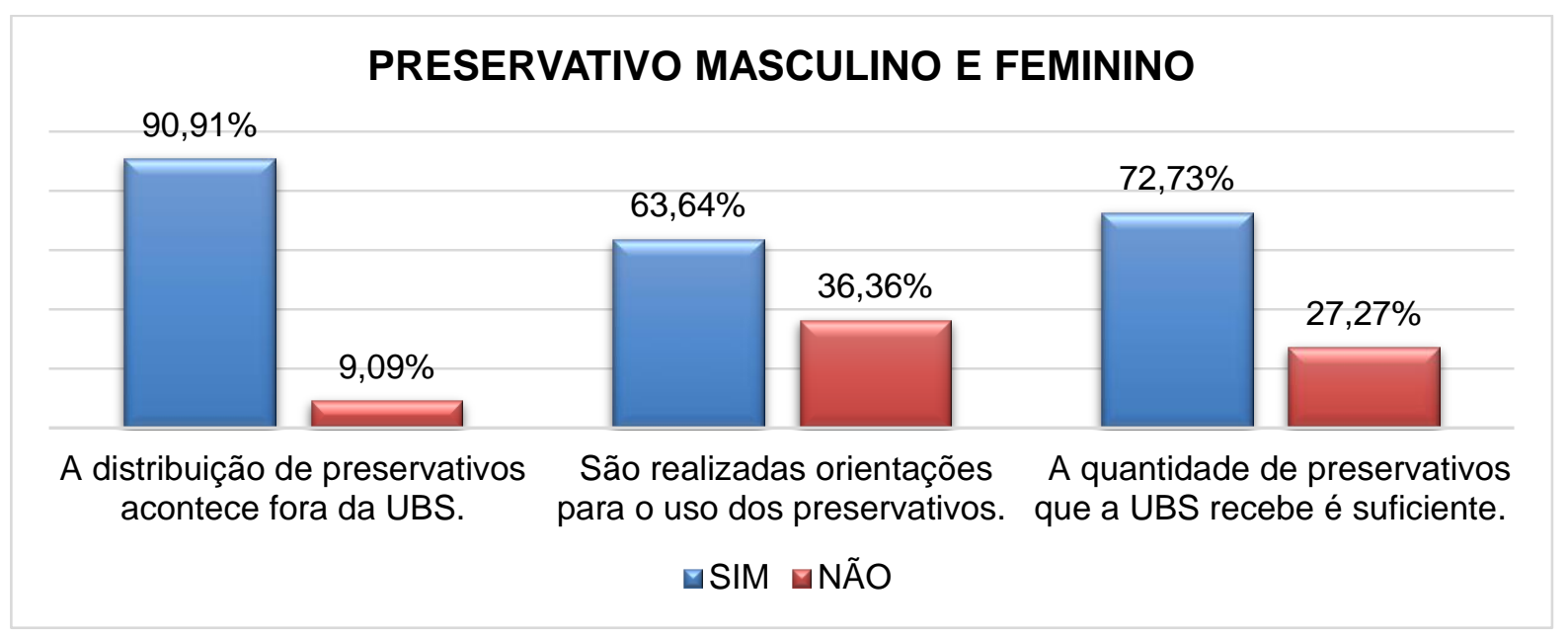

Fonte: Elaboração própria das autoras com base em dados coletados em pesquisa (2019).

No que diz respeito ao suprimento de preservativos para atender as necessidades da população, $72,73 \%$ dos pesquisados afirmaram que o quantitativo ofertado a UBS é satisfatório; enquanto $27,27 \%$ responderam não obter em quantidade necessária.

A distribuição dos preservativos para a população é importante para incentivar a prevenção do HIVIAIDS, bem como de outras IST's. Porém, se faz necessário a realização de atividades educativas que esclareçam as possíveis dúvidas e orientem as pessoas sobre a utilização do preservativo nas relações sexuais. ${ }^{30}$

Os soropositivos apresentam predisposição a terem prejuízos físicos e psicológicos, associados à rejeição, distinção e segregação pela sociedade. Desta forma, esses indivíduos enfrentam dificuldades quanto à aceitação da descoberta da patologia e com relação ao preconceito da população. ${ }^{12}$

O temor e a falta de conhecimento ampliado quanto à contaminação pelo HIV/AIDS, leva os profissionais da saúde a se sentirem despreparados para prestarem assistência aos pacientes infectados. Por isso, ocasionalmente identifica - se casos de preconceito dos profissionais direcionado aos pacientes soropositivos, por receio de se contaminar durante a realização do atendimento. ${ }^{14}$

No que tange a assistência aos pacientes soropositivos, $100 \%$ dos participantes da pesquisa afirmaram que a UBS realiza o acompanhamento desses pacientes. Quanto a referenciar os pacientes portadores da infecção pelo HIV, a maioria dos profissionais de 
enfermagem pesquisados $(90,91 \%)$ afirmaram que a UBS realiza esse referenciamento (Gráfico 5).

Gráfico 5. A assistência de enfermagem as pessoas diagnosticadas com HIV/AIDS.

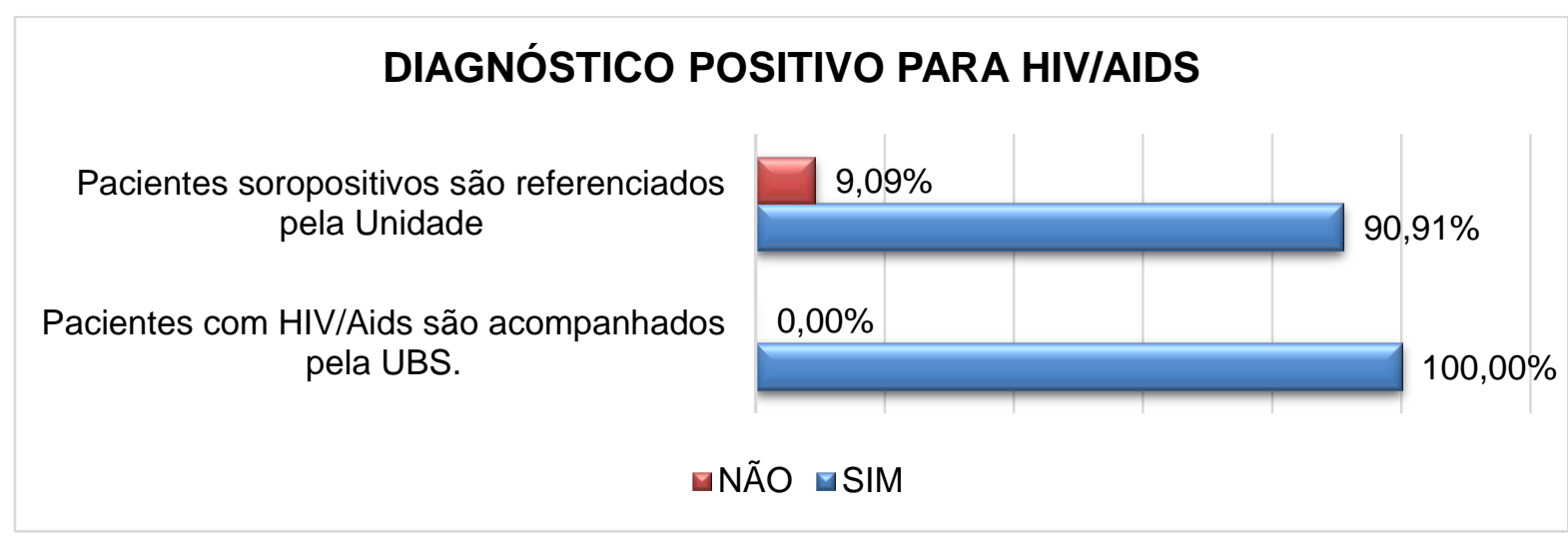

Fonte: Elaboração própria das autoras com base em dados coletados em pesquisa (2019).

É essencial que após o diagnóstico positivo para o HIV, ocorra o acompanhamento clínico do paciente, pois a partir da detecção se faz necessária à realização de uma série de cuidados e orientações com o intuito de proporcionar uma assistência eficaz e satisfatória aos pacientes soropositivos, assim como possibilitar uma melhor qualidade de vida ao portador da infecção. ${ }^{30}$

$\mathrm{O}$ cuidar em enfermagem compreende $\mathrm{o}$ ato de repassar dedicação e empenho de um indivíduo para o outro, objetivando auxiliar, salvaguardar e conservar a sociedade, contribuindo para que os seres humanos identifiquem sentido na patologia, na tristeza e na dor. É também apoiar um indivíduo na aquisição de novas informações e do autocontrole. $^{22}$

Todos os integrantes da pesquisa confirmaram que é realizada a solicitação do exame sorológico para o HIV durante todas as primeiras consultas de pré-natal. No que se refere à revelação do resultado do $1^{\circ}$ e $2^{\circ}$ exame sorológico para o HIV, os participantes foram questionados se essa revelação ocorre ainda no período da gestação. Novamente $100 \%$ dos profissionais responderam "sim".

É fundamental que o enfermeiro realize a solicitação de exames no pré-natal, preconizados pelo MS, uma vez que é por meio deles que poderão ser detectadas patologias ou alterações homeostáticas que merecem especial atenção. Convém lembrar que, dentre esses exames que devem ser realizados, estão os testes rápidos e sorológico 
para o diagnóstico da infecção pelo HIV, permitindo a intervenção prévia e consequente prevenção de contaminação fetal ou transmissão vertical. ${ }^{31}$

Em referência à solicitação do teste rápido ou sorológico para o HIV, 54,55\% dos profissionais integrantes da pesquisa afirmaram realizar essa solicitação as pacientes que relatam sinais e sintomas que podem estar relacionados a presença de infecções ginecológicas. Em contradição, $45,45 \%$ dos participantes afirmaram não realizar a referida solicitação. Os testes rápidos e sorologias devem estar disponíveis nos serviços de saúde para qualquer usuário que tenha se exposto ao vírus, e não somente quando apresentar sinais e sintomas.

Nos últimos tempos esta patologia obteve uma incidência significativa, sendo classificada como um problema de saúde pública. No entanto, a doença ainda tem provocado repercussão entre os indivíduos portadores desta doença, devido a discriminação e o preconceito, o que pode ocasionar problemas psíquicos, desestruturação familiar e alterações no convívio social. ${ }^{13}$

Gráfico 6. A solicitação e realização de teste rápido ou sorológico para o HIV.

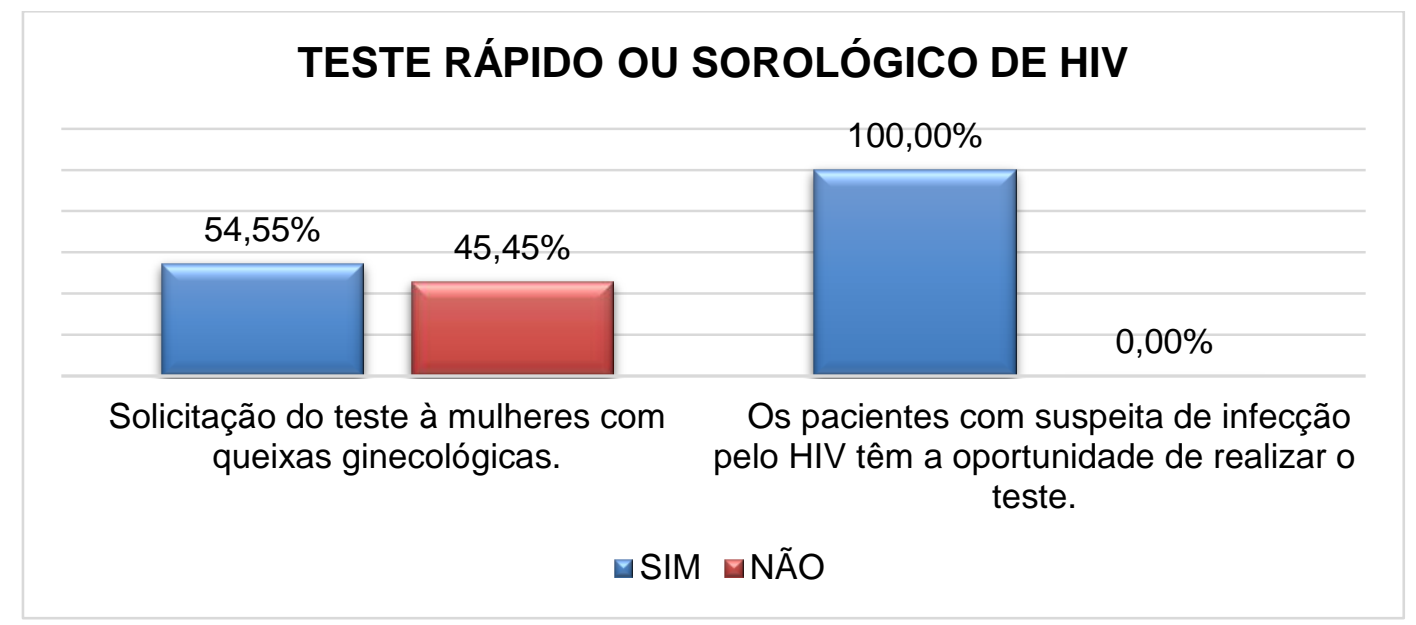

Fonte: Elaboração própria das autoras com base em dados coletados em pesquisa (2019).

Quanto aos pacientes que vão ao estabelecimento de Saúde com suspeita de contaminação pelo HIV, 100\% dos enfermeiros e técnicos de enfermagem pesquisados afirmaram que é assegurado o direito dessas pessoas de realizarem os testes rápidos (Gráfico 6).

A Atenção Primária a Saúde possui função primordial na prevenção e detecção da infecção pelo HIV, pois durante a prestação da assistência podem ser solicitados e realizados testes rápidos ou sorológicos nos pacientes com sinais e sintomas sugestivos 
de contaminação pelo vírus. Isso contribui para o diagnóstico precoce e para a redução da morbidade e mortalidade da população. ${ }^{32}$

Tendo em consideração a disponibilidade de insumos para a execução de testes rápidos para o HIV/AIDS, $100 \%$ dos participantes da pesquisa afirmaram que a UBS dispõe os insumos necessários para realizar os testes. Porém, quanto a oferta de teste rápido ou sorológico para o HIV aos pacientes da Unidade Básica de Saúde em estudo; $90,91 \%$ afirmaram que a UBS oferece os testes, enquanto $9,09 \%$ afirmaram que não (Gráfico 7).

Tendo em vista que os profissionais de enfermagem devem desenvolver o aconselhamento pré e pós-teste rápido para o HIV, 63,64\% dos pesquisados afirmaram executar o aconselhamento, porém 36,36\% disseram não realizar (Gráfico 7).

Gráfico 7. A realização de testes rápidos para o HIV na Atenção Primária a Saúde.

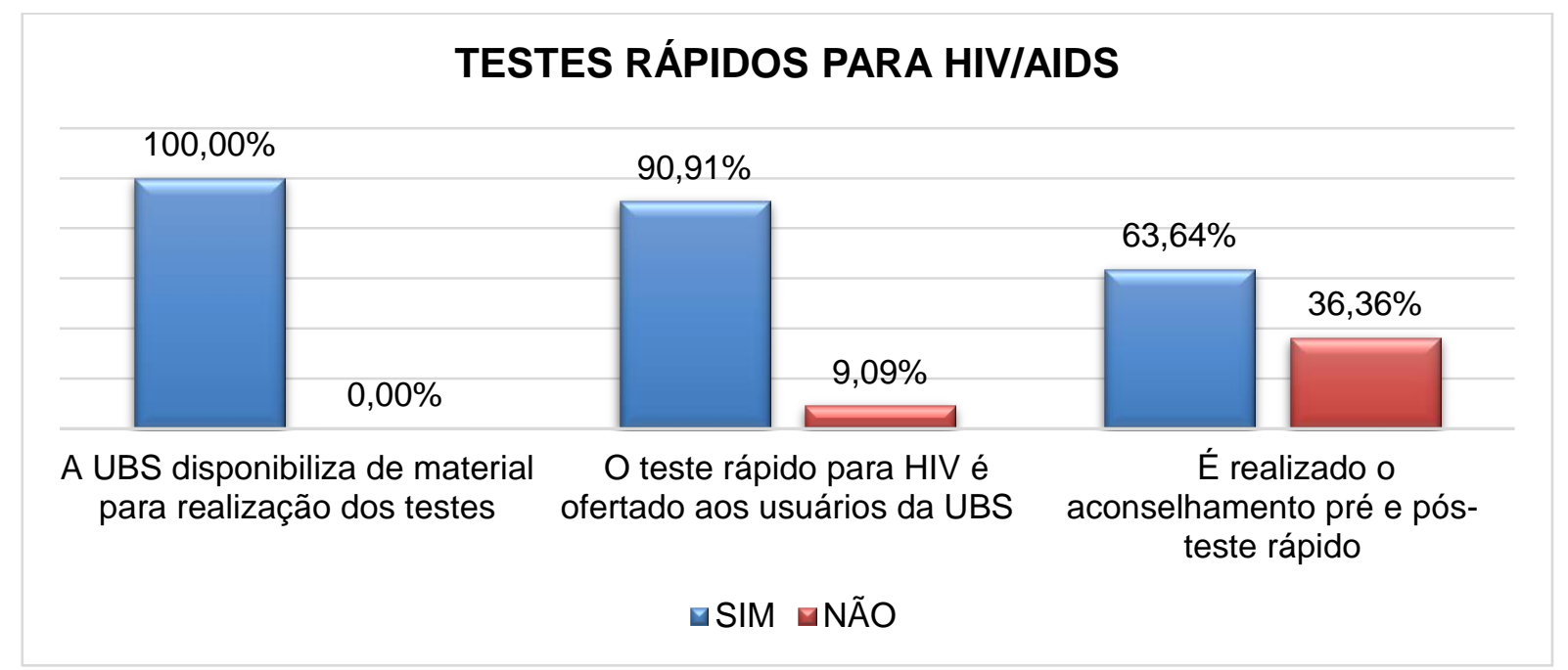

Fonte: Elaboração própria das autoras com base em dados coletados em pesquisa (2019).

A detecção precoce do HIV é de suma importância para a qualidade de vida do paciente soropositivo. Para auxiliar nisso, foram desenvolvidas inovações no âmbito do diagnóstico da infecção pelo HIV, pois foram criados os testes rápidos que podem ser definidos como imunonsaios, de execução simples, rápida e eficaz. Além desses benefícios, convém lembrar que, durante a realização do procedimento deve ser executado o aconselhamento pré e pós-teste, com a finalidade de orientar e esclarecer as dúvidas do paciente. Esses testes proporcionaram uma maior possibilidade de acesso da sociedade ao diagnóstico do HIV. ${ }^{19,30}$ 
Portanto, na consulta de enfermagem é extremamente importante o foco na escuta do paciente, pois a mesma permite o desenvolvimento de um vínculo subsidiado por segurança, credibilidade, admiração e empatia. Isso contribui para que o indivíduo se sinta confortável para expressar seus medos, aflições, preocupações e dúvidas; aspectos essenciais para que o tratamento seja consolidado de forma apropriada. ${ }^{33}$

Dos enfermeiros e técnicos de enfermagem que participaram da pesquisa, 45,45\% afirmaram ter participado de treinamento para a execução dos testes rápidos para o HIV, entretanto, $54,55 \%$ disseram não ter participado de nenhuma capacitação com esta temática.

A atualização dos profissionais de enfermagem é extremamente importante para que seja ofertada uma assistência eficaz aos pacientes, uma vez que, é através de educação permanente que os profissionais poderão aprimorar seus conhecimentos e adquirir novas informações. ${ }^{26}$

Para a realização dos testes rápidos também se faz necessária à capacitação dos profissionais de enfermagem, pois é por meio do treinamento que os enfermeiros poderão adquirir o conhecimento necessário para a execução do procedimento com eficácia. ${ }^{26,32}$

O método de educação em saúde pode auxiliar no alcance de informações que auxiliam a minimizar a reação negativa do indivíduo quanto ao HIV/AIDS, exigindo do enfermeiro estar perto da vivência cotidiana dessas pessoas, realizando uma análise relacionada à sua função de provedor do conhecimento em saúde e elaborando espaços direcionados a aquisição de instruções por essas pessoas. ${ }^{16,17}$

Quanto ao desenvolvimento de atividades de educação em saúde direcionadas aos usuários da Unidade Básica de Saúde sobre as medidas de prevenção das IST's; $81,82 \%$ dos profissionais pesquisados afirmaram executar atividades educativas sobre 0 assunto, por outro lado $18,18 \%$ disseram não desenvolver ações sobre o tema (Gráfico 8). Quando questionados sobre quais seriam essas ações educativas realizadas, a maioria dos que disseram sim, citaram "palestras nas escolas e nos setores da cidade".

Com relação à efetuação de atividades de educação relacionadas a profilaxia das IST's no estabelecimento de saúde pesquisado; $81,82 \%$ dos participantes da pesquisa afirmaram desenvolver atividades sobre essa temática na UBS, enquanto $18,18 \%$ disseram não realizar (Gráfico 8). 
Gráfico 8. Ações educativas para a prevenção das infecções sexualmente transmissíveis (IST's).

\section{PREVENÇÃO DAS INFECÇÕES SEXUALMENTE TRANSMISSÍVEIS - IST'S}

São realizadas ações educativas no espaço físico da UBS.

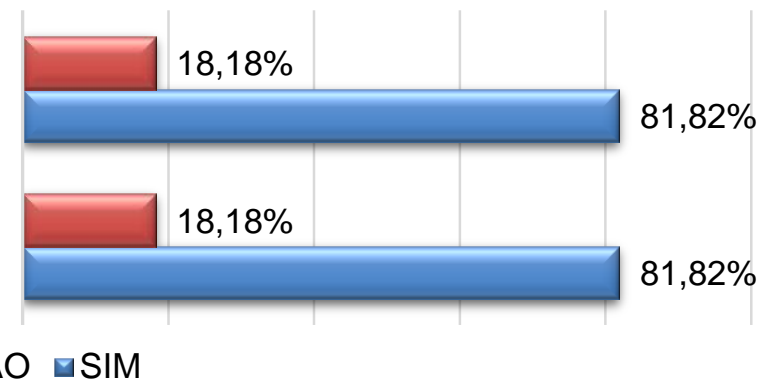

Fonte: Elaboração própria das autoras com base em dados coletados em pesquisa (2019).

É essencial que o enfermeiro esteja preparado para realizar campanhas educativas para a população, tanto dentro do espaço físico da Unidade, quanto na área de cobertura da UBS; pois o enfermeiro possui um papel fundamental na transmissão de conhecimentos e conscientização da sociedade. Um tema extremamente relevante para a realização dessas ações educativas, está relacionado a prevenção das infecções sexualmente transmissíveis, pois se trata de um problema de saúde pública que merece especial atenção por parte dos profissionais de enfermagem. ${ }^{34}$

Dentre os sujeitos da pesquisa, 100\% afirmaram fazer a notificação de IST's no Sistema de Informação de Agravos de Notificação (SINAN).

Nesse contexto, o vínculo de confiança entre usuário, família e enfermeiro é fundamental para a aceitação do recurso terapêutico, que é primordial para o paciente soropositivo. É necessário que o enfermeiro esteja apto ao revelar o diagnóstico e as suas possíveis consequências, pois neste momento é possível interferir de maneira negativa no emocional e no tratamento do usuário, bem como o elo entre o profissional e o paciente. Convém lembrar que a revelação do diagnóstico de HIV/AIDS deve ser norteada por muita atenção, cautela e sabedoria. ${ }^{15,18}$

A notificação dos casos de IST's deve ser obrigatoriamente realizada no SINAN, que possui o intuito de processar e organizar dados sobre as patologias e agravos de notificação. Essas notificações precisam ser desenvolvidas de forma adequada e regular, possibilitando que os dados sejam empregados na escolha de possíveis ações de vigilância. Pois a partir do diagnóstico positivo da IST e a execução da notificação, será 
possível iniciar o tratamento prescrito, bem como realizar a busca ativa dos parceiros sexuais das pessoas portadoras da infecção. ${ }^{29,35}$

Entre os participantes da pesquisa, 90,91\% dos profissionais pesquisados afirmaram executar a busca ativa dos usuários que foram diagnosticados com HIV, porém não voltaram a Unidade Básica de Saúde para buscar o resultado. Por outro lado, 9,09\% dos pesquisados disseram não realizar essa busca ativa.

Após um diagnóstico positivo para o HIV, surgem múltiplas responsabilidades para o enfermeiro, uma vez que este possui função primordial na realização da busca ativa do paciente soropositivo e de seus parceiros sexuais, bem como na prestação de cuidados e orientações. A partir da detecção, se faz necessário iniciar um acompanhamento detalhado e eficiente do paciente infectado, com a finalidade de verificar a adesão do usuário ao tratamento e ofertar informações essenciais para auxilialo no convívio com a nova situação de saúde. ${ }^{36}$

Em geral, os indivíduos que convivem com HIV/AIDS são prejudicados fisicamente e psicologicamente. A descoberta do diagnóstico pode gerar desespero, angústia, inquietação, preocupação e receio da discriminação, do preconceito da sociedade, da progressão da patologia e da morte. ${ }^{22}$

Diante de um paciente portador de HIV/AIDS, o atendimento da enfermagem realmente se torna mais difícil, uma vez que se faz necessária a elaboração de estratégias para fornecer uma atenção à saúde que transforme a realidade, agindo nos aspectos significativos e promovendo o cuidado físico e psíquico de forma holística e eficiente. É importante salientar que o papel fundamental da enfermagem não é exatamente a cura, mas sim uma conduta que inclua atuações e estratégias que busquem reduzir o sofrimento do paciente, preservar a integridade e proporcionar métodos para atuar diante de impasses e riscos. Para realizar o cuidado é primordial possuir informações qualificadas para nortear o exercício do enfermeiro. ${ }^{21}$

\section{CONSIDERAÇÓES FINAIS}

Em virtude dos fatos mencionados, entende-se que os enfermeiros e técnicos de enfermagem da Atenção Primária a Saúde (APS) apresentam importância tanto na prevenção da infecção pelo HIV, quanto na prestação da assistência aos pacientes soropositivos. 
Identificamos por meio dos dados coletados, que ainda existe uma carência significativa de conhecimentos sobre o HIV/AIDS, por parte dos enfermeiros e técnicos de enfermagem; evidenciando a necessidade destes profissionais receberem atualizações relacionadas a temática. Percebe-se, através da pesquisa, que ainda existem obstáculos para realização de ações de educação em saúde voltadas para a conscientização da população sobre a prevenção da infecção pelo HIV.

Ainda convém lembrar que, para o desenvolvimento desta pesquisa, existiram dificuldades principalmente no que se refere a resistência de outras secretarias de saúde a aceitarem a realização da pesquisa com os profissionais de enfermagem de seu município, alegando se tratar de um tema "polêmico". Essa resistência comprovou ainda mais a necessidade do desenvolvimento de novos estudos sobre o assunto, com o intuito de ampliar os conhecimentos da população e identificar a realidade de outros municípios.

\section{REFERÉNCIAS}

1 Silveira JA, Sousa CS, Lima AGA, Silva BC e Araújo LS. Processo de Enfermagem aplicado ao paciente soropositivo: Relato de experiência. Anais do Congresso Internacional de Enfermagem. UNIT, Sergipe; 9-12 maio 2017. Disponível em: https://blog.mettzer.com/formatacao-normasvancouverl. Acesso em: 04/03/2019.

2 Campelo CL, Silva AA, Sousa AMM, Rolim ILTP, Aquino DMC. Assistência de Enfermagem Frente à Criança Portadora do Vírus HIV: Uma Revisão Integrativa da Literatura. Revista UNINGÁ. Vol.51, pp.52 - 57; Jan - Mar 2017. Disponível em: http://revista.uninga.br/index.php/uninga/article/view/1334/952 . Acesso em: 04/03/2019.

3 Villarinho MV, Padilha MI. Sentimentos Relatados pelos Trabalhadores da Saúde Frente à Epidemia da AIDS (1986-2006). Texto Contexto Enfermagem, 2016; 25 (1): e0010013. Disponível em: http://www.scielo.br/pdf/tce/v25n1/0104-0707-tce-25-01-0010013.pdf. Acesso em: 04/03/2019.

4 Almeida Junior JA, Moraes AAS, Barreto MASA, Santos FS, Suto CSS e Paiva LBF. Teste Rápido Para HIV: Representações Sociais de Profissionais da Atenção Básica. Revista Baiana de Enfermagem. $\quad$ v32, (2018). Disponível em: https://rigs.ufba.br/index.php/enfermagem/article/view/25885/16489. Acesso em: 19/04/ 2019.

5 Garbin CAS, Sandre AS, Rovida TAS, Pacheco KTS, Pacheco Filho AC e Garbin AJl. O Cuidado Para Pessoas com HIV/AIDS Sob a Ótica De Agentes Comunitários de Saúde. Trabalho Educação e Saúde, Rio de Janeiro, 2019. Disponível em: http://www.scielo.br/pdf/tes/v17n1/01026909-tes-17-1-e0018508.pdf. Acesso em: 19/04/2019.

6 Conselho Federal de Enfermagem. Resolução n. 564/2017: Código de Ética dos Profissionais de Enfermagem. Disponível em: http://www.cofen.gov.br/resolucao-cofen-no-5642017 59145.html . Acesso em: 08/03/2019. 
7 Fontinele DCSS, Pereira FCC, Rocha KMM, Silveira Júnior LS, Santos NRO. Ações de Enfermagem para Inserção dos Homossexuais Soropositivos nos Serviços de Saúde. Revista Humano Ser - UNIFACEX, Natal-RN, v.3, n.1, p. 84-100, 2017/2018. Disponível em: https://periodicos.unifacex.com.br/humanoser/article/view/1006/326 . Acesso em: 05/03/2019.

8 Maciel KL, Milbrath VM, Freitag VL, Gabatz RIB, Silva MS e Santos BA. Estratégias de Assistência no Cuidado à Pessoa com Síndrome da Imunodeficiência Adquirida. Revista Enfermagem Atual, 2018. Disponível em: https://www.revistaenfermagematual.com.br/index.php/revista/article/view/133/45. Acesso em: 19/04/2019.

9 Silva AG, Cavalcanti VS, Santos TS, Bragagnollo GR, Santos KS e Santos IMS. Revisão integrativa da literatura: cuidados de enfermagem a idosos com HIV. Rev. Bras. Enferm. vol.71 supl.2. Brasília, 2018. Disponível em: http://www.scielo.br/scielo.php?script=sci arttext\&pid=S003471672018000800884\&lang=pt Acesso em: 08/03/2019.

10 Silva RA, Castro RR, Pereira IR, Oliveira SS. Questionário para avaliação das ações de controle do HIV/Aids na Atenção Básica. Acta Paulista de Enfermagem, [online], vol.30, n.3, 29 de maio de 2017, pp.271-279. Disponível em: http://www.scielo.br/scielo.php?script=sci abstract\&pid=S010321002017000300271\&lng=en\&nrm $=$ iso\&tlng=pt. Acesso em 20/04/2019.

11 Lima MCD. O Serviço Social e a Adesão ao Tratamento de Crianças e Adolescentes Soropositivos: Relato de Experiência. Divers@ Revista Eletrônica Interdisciplinar, Matinhos, v.8, n. 1, p. 43-50, jan./jun. 2015. Disponível em: https://revistas.ufpr.br/diver/article/view/43876/26610. Acesso em: 16/04/2019.

12 Albuquerque JR, Batista AT e Saldanha AAW. O Fenômeno do Preconceito nos Relacionamentos Sorodiferentes para o HIV/AIDS. Psicologia, Saúde \& Doenças, vol. 19 n.ํㅜ 2, p. 405-421. Lisboa, ago. 2018. Disponível em: http://www.scielo.mec.pt/scielo.php?script=sci arttext\&pid=S164500862018000200019. Acesso em: 16/04/2019.

13 Santos WN. Análise de Conceito do Diagnóstico de Enfermagem Controle Ineficaz da Saúde em Pessoas Vivendo com AIDS. Natal. Dissertação (Mestrado em Enfermagem) - Universidade Federal do Rio Grande do Norte, Centro de Ciências da Saúde, Programa de Pós-Graduação em Enfermagem; $2018 . \quad$ Disponível em: https://repositorio.ufrn.br/jspui/bitstream/123456789/26455/1/An\%C3\%A1liseconceitodiagn\%C3\% B3stico Santos 2018.pdf. Acesso em: 20/04/2019.

14 Garbin CAS, Martins RJ, Belila NM e Garbin AJl. O estigma de usuários do sistema público de saúde brasileiro em relação a indivíduos HIV positivo. DST - J bras. Doenças Sexualmente Transmissíveis, p.12-16, 2017. Disponível em: http://www.dst.uff.br/revista29-12017/DST\%20v29n1 12-16 PT.pdf. Acesso em: 17/04/2019.

15 Bezerra VP, Serra MAP, Almeida SA, Pereira IL, Chaves RB e Nogueira JA. Ações de Prevenção do HIV e de Promoção à Saúde no Contexto da AIDS pela Estratégia Saúde da Família em João Pessoa-PB. Ciência, Cuidado e Saúde, Abr./Jun. 2016; p. 343-349. Disponível em: http://ojs.uem.br/ojs/index.php/CiencCuidSaude/article/view/28900/pdf. Acesso em: 21/04/2019. 
16 Pinto ACS, Queiroz MVO, Gubert FA, Braga VAB e Pinheiro PNC. Educação Em Saúde na Prevenção do HIV/AIDS com Homens Jovens Usuários de Crack. Texto Contexto Enfermagem, 2016. Disponível em: https://www.redalyc.org/pdf/714/71446759026.pdf. Acesso em: 20/04/2019.

17 Ramos FBP, Carvalho IM , Silva Filho WP , Nunes PS e Nóbrega MM. A Educação em Saúde como Ferramenta Estratégica no Desenvolvimento de Ações de Prevenção da Transmissão do HIV: Um Relato de Experiência. Revista Eletrônica Acervo Saúde. Electronic Journal Collection Health. Vol.Sup.19. 2019. Disponível em: file:///C:/Users/Aluno\%2004/Downloads/509-Artigo-15501-10-20190317.pdf. Acesso em: 20/04/2019.

18 Costa GM, Figueredo RC e Ribeiro MS. A Importância do Enfermeiro Junto ao PSE nas Ações de Educação em Saúde em uma Escola Municipal de Gurupi - TO. Revista Científica do ITPAC, Araguaína, v.6, n2, Pub.6, Abril 2013. Disponível em: https://assets.itpac.br/arquivos/Revista/62/6.pdf . Acessado em 01/05/2019.

19 Silva ITS, Valença CN, Silva RAR. Cartografia da Implementação do Teste Rápido Anti-HIV na Estratégia Saúde da Família: Perspectiva de Enfermeiros. Escola Anna Nery. Revista de Enfermagem, vol. 21, núm. 4, 2017, pp. 1-8. Rio de Janeiro, 2017. Disponível em: http://www.scielo.br/pdf/ean/v21n4/pt 1414-8145-ean-2177-9465-EAN-2017-0019.pdf Acesso em: 05/05/2019.

20 Massignani LRM, Rabuske MM, Backes MS \& Crepaldi MA. Comunicação de Diagnóstico de Soropositividade HIV e AIDS por Profissionais de Saúde. Psicologia Argumento, Curitiba, v. 32, n. 79, p. 65-75, Supl. 1 , 2014. Disponível https://periodicos.pucpr.br/index.php/psicologiaargumento/article/view/20367/19635 Acesso em: 08/05/2019.

21 Silva JO e Valente GSC. O Enfermeiro de Saúde Coletiva no Tratamento e Acompanhamento do Idoso Soropositivo. Revista Enfermagem Atual, p. 19-26, 2017. Disponível em: http://revistaenfermagematual.com/arquivos/ED 82 REVISTA 20/02.pdf. Acesso em: 10/05/2019.

22 Isoldi DMR; Carvalho FPB; Simpson CA. Análise contextual da assistência de enfermagem à pessoa com HIV/AIDS. Revista Online de Pesquisa Cuidado Fundamental. Universidade Federal do Estado do Rio de Janeiro. Escola de Enfermagem Alfredo Pinto. V. 9, n. 1, p. 273-278, jan./mar., $2017 . \quad$ Disponível em: http://www.seer.unirio.br/index.php/cuidadofundamental/article/view/4119/pdf 1 . Acesso em 10/05/2019.

23 COFEN - Conselho Federal de Enfermagem. Pesquisa Inédita Traça Perfil Da Enfermagem. 2015. Disponível em: http://www.cofen.gov.br/pesquisa-inedita-traca-perfil-daenfermagem 31258.html. Acessado em: 10/10/2019.

24 Araujo MAN de Lunardi Filho WD, Alvarenga MRM et al. Perfil Sociodemográfico dos Enfermeiros da Rede Hospitalar. Rev. Enferm. UFPE on line., Recife, nov., 2017. P. 4716-4725. Disponível em: https://pdfs.semanticscholar.org/ef0b/50d0f0ebe1cabd903080f3b67fc1f920b3e6.pdf. Acesso em 11/10/2019.

25 Machado $\mathrm{MH}$, Aguiar Filho W, Lacerda WF, Oliveira E, Lemos W, Wermelinger $M$, et. al. Características Gerais Da Enfermagem: O Perfil Sócio Demográfico. Enferm. Foco, 2016. Disponível em: $\quad$ http://revista.cofen.gov.br/index.php/enfermagem/article/view/686/296. Acesso em: 12/10/2019. 
26 Marcondes FL, Tavares CMM, Santos GS, Silva TN, Silveira PG. Capacitação Profssional de Enfermagem na Atenção Primária à Saúde: Revisão Integrativa. Revista Pró Univer SUS. 2015 Jul./Dez. $\quad$ p. 09-15. $\quad$ Disponível em: https://www.researchgate.net/publication/317951885 Capacitacao profissional de enfermagem na atencao primaria a saude revisao integrativa . Acesso em: 14/10/2019.

27 Portinari DB, Wolfgang SMBM. Imagens e Marcas: Um Imaginário Ligado à Epidemia de HIVAids no Brasil. ALCEU - v. 17 - n.34 - p. 45 a 60 - jan./jun. 2017. Disponível em: http://revistaalceuacervo.com.puc-rio.br/media/alceu34 pp45-60.pdf.Acesso em: 15/10/2019.

28 Dias CA, Alves JH, Leão LO, Barroso NC, Avanzi SA, et al. Programa de humanização do prénatal e nascimento: reflexões sobre a efetividade das estratégias de redução da transmissão vertical do HIV. - Anais, 2019. Disponível em: http://www.abep.org.br/xxiencontro/arquivos/R02271.pdf . Acesso em: 16/10/2019.

29 Cavalcante EGF, Miranda MCC, Carvalho AZFHT, Lima ICV, Galvão MTG. Notificação de Parceiros Sexuais com Infecção Sexualmente Transmissível e Percepções dos Notificados. Rev Esc Enferm USP . 2016; p. 450-457. Disponível em: http://www.scielo.br/pdf/reeusp/v50n3/pt 0080-6234-reeusp-50-03-0450.pdf . Acesso em: 17/10/2019.

30 Ramos FBP, Carvalho IM, Silva Filho WP, Nunes PS, Nóbrega MM. A educação em Saúde como Ferramenta Estratégica no Desenvolvimento de Ações de Prevenção da Transmissão do HIV: Um Relato de Experiência. Revista Eletrônica Acervo Saúde / Electronic Journal Collection Health. Vol.Sup.19, 2019. Disponível em: https://www.acervomais.com.br/index.php/saude/article/view/509/307 Acesso em 17/10/2019.

31 Ferreira BCS; Almeida NMS. O Conhecimento das Gestantes Acerca das Sorologias Solicitadas Durante o Pré-Natal. Id on Line Rev. Psic. V.11, N. 35. Maio/2017. Disponível em: https://idonline.emnuvens.com.br/id/article/view/723/1010. Acesso em 20/10/2019.

32 Araújo WJ, Quirino EMB, Pinho CM, Andrade MS. Percepção de Enfermeiros Executores de Teste Rápido em Unidades Básicas de Saúde. Rev Bras Enferm [Internet]. 2018; p. 676-81. Disponível em: http://www.scielo.br/pdf/reben/v71s1/pt 0034-7167-reben-71-s1-0631.pdf . Acesso em: 20/10/2019.

33 Macêdo SM, Sena MCS e Miranda KCL. Consulta de Enfermagem ao Paciente com HIV: Perspectivas e Desafios Sob a Ótica de Enfermeiros. Revista Brasileira de Enfermagem, Brasília, mar-abr de 2013, p. 196-201. Disponível em: https://www.redalyc.org/html/2670/267028666007/. Acesso em: 25/10/2019.

34 Santos AHF, Souza DM, Souza LS e Lobo MRG. Atuação do Enfermeiro na Prevenção de Infecções Sexualmente Transmissíveis na Atenção Básica. v. 1 n. 1: Anais dos CONCIFA, 2018. Disponível em: http://periodicos.fametro.edu.br/index.php/AE/article/view/22 . Acesso em 25/10/2019.

35 Melo MAS, Coleta MFD, Coleta JAD, Bezerra JCB, Castro AM, Melo ALS, et al. Percepção dos profissionais de saúde sobre os fatores associados à subnotificação no Sistema Nacional de Agravos de Notificação. Rev. Adm. Saúde - Vol. 18, № 71, abr. - jun. 2018. Disponível em: http://cah.org.br/ojs-2.4.8/index.php/ras/article/view/104/153 . Acesso em: 20/10/2019.

36 Silva RAR, Costa MMN, Souza Neto VL, Silva BCO, Costa CS, Andrade IFC. Falta de Adesão em Pessoas Vivendo com HIV: Acurácia das Características Definidoras do Diagnóstico de 
DOI: 10.18606/2318-1419/amazonia.sci.health.v8n2p2-23

Revista Amazônia Science \& Health

ISSN: 2318-1419

2020, Vol. 8 , № 2
FEITOZA, LML. ROSA, SRS. SILVA, LS. FIGUEIREDO, RC. SILVA, RS

Principais características da atuação dos profissionais de enfermagem da atenção primária em saúde na assistência ao paciente soropositivo.

Enfermagem. Revista Latino-Americana de Enfermagem, Universidade de São Paulo, Ribeirão Preto. vol. 25, 2017, p. 1-10. Disponível em: https://www.redalyc.org/pdf/2814/281449566093.pdf . Acesso em 30/10/2019. 